\title{
The Effect of Active Failure on the Power System Reliability Evaluation \\ ${ }^{1, a}$ Yuxin Zhao, ${ }^{2, b}$ Xiaohui $\mathrm{Hu},{ }^{3, \mathrm{c}}$ Zhao Sun
}

Tianjin Electric Power Corporation Electrical Power Research Institute,Tianjin ,China

ayx_zhao1101@163.com

Keywords: Reliability, Power System, Active Failure.

\begin{abstract}
The active failure is important to the power system reliability. The effect of the active failure is proposed and analyzed to evaluate the power system reliability by the amending parameter method and the simulating failure method. in this paper. The need of the considering active failure is verified by the analysis of the reliability evaluation of the IEEE-RTS system, the most serious fault state and the system weaknesses evaluated by the methods above. The analysis of the IEEE-RTS system indicates that the effect of the active failure on the system reliability of is effective and applied.
\end{abstract}

\section{Introduction}

In recent years, worldwide power blackouts occurred in many cities at home and abroad, such as "8·14" Blackout in USA \& Canada [1,2] which caused enormous damage to society. The Frequent occurrences of the blackouts make the world deeply aware of the importance of the reliability of power system and pay more attention to the power system reliability. In order to avoid the occurrence of worldwide power blackout accident, we must put more manpower and material resources for research on power system reliability.

It can be found that the blackouts are often caused by the common mode failure which is often caused by the active failure [3] from theanalysis of the serious blackouts. However, components of the system are assumed to be independent of each other, the power plant or substation main wiring is simplified for a node, and the main wiring components (such as circuit breaker, bus, relay protection element) fault caused by power generation unit or line outage is often ignored which are important to the power system reliability evaluation. Actually, the main wiring component failure, especially the active failure can be regarded as the causes of the blackout which has a more serious consequence. So, it is important to analyze the effect of active failure on the power system reliability.

\section{The Analysis of the Active Failure}

Reliability Model[6].The active failure is a failure mode which causes the protective action, and causes the other components to be in a failure when a single component fault. The active failure often causes the common mode failure which is regarded the main cause of the worldwide blackout.

Some components are generally considered as the three state models which are the normal operation state, the fault repair state and the planned maintenance state. When the compoment itself is out of operation, system state has no obvious change, or the fault clearing process is very short. These components can be ignored the effect of the active failure. But there are also a kind of components such as circuit breakers, which have complex structure, many actions, and various fault types including the active failure. Once the active failure of this type component occurs, its own actions can not clear the failure, which need the backup protection or adjacent component protection to clear. These components need to consider the effect of the active failure on the power system reliability. These components mainly include circuit breakers, bus and relay protection components.

The action of relay protection is ultimately reflected in the circuit breaker. Therefore, the effect of the relay protection[4,5] is credited to the reliability model of the circuit breaker by analyzing the relay protection action and protection area. The misoperation of the relay protection is similar to the 
state $\mathrm{S}$ of the circuit breaker. So the rate of the misoperation of the relay protection is added to the rate of state $S$ of the circuit breaker to reflected in the effect. The consequence of the refusing action of the relay protection is equivalent to the active failure of the all circuit breakers which are in the protection area of the relay protection. The consequence is more serous than that of the state $\mathrm{S}$. We call it state F. The reliability model of the active failure is shown in Fig 1.

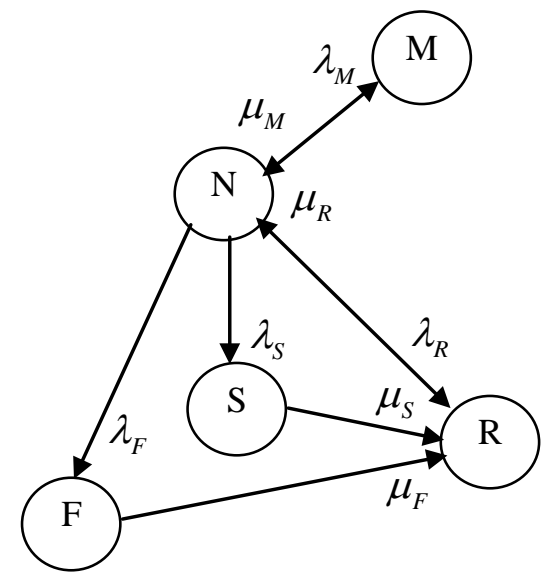

Fig 1 the reliability model of the relay protection

The Active Failure Analysis. The component with the active failure influences the states of the power system components through their electrical path in the main wiring. Therefore, the analysis of the active failure depends on the analysis of the location of the components in the main wiring and the analysis of the protection range of the components.

When the active failure of a component occurs, it will make those elements trip which make the component with active failure isolated from the main wiring. Therefore, it is needed to find out a set of components that make the components with active failure isolated from the main wiring. The set is called the protection range of the component. To search the network can find the protection range of the component.

In order to illustrate the range of the active failure better, we calculate the path set controlled by the components with the active failure. So, when a component occur the active failure, it can be clearly known the path cut off, and thereby known the influence range of the component.

Under normal circumstances, there are PS paths in the network. In the set of components controlled by PS bits in computer memory, the value 1 of the digits represents the minimum path set. When the PS is greater than the computer bits, the paths can be resented by more than one bit.

Assuming $R(i)$ represents the path set of the component $i$. When the component $i$ falls in outage, the remaining path set of the main wiring is calculated by the Eq.1.

$$
X=L \backslash R(i)=\overline{R(i)}
$$

When the set of the components $\lambda_{F}$ is in outage, $\bigcup_{i \in \xi} R(i)$ is expressed the path set which is cut off, and $L \backslash \bigcup_{i \in \xi} R(i)$ is expressed the remained path set. When earth fault or short circuit fault occurs, the circuit breakers in the protection range of the component should trip. The set $I$ is expressed the breaker set in the protection range of the component $i$. So when the active failure of the component $i$ occurs, the path set cut off is described as the Eq.2.

$$
S(i)=\bigcup_{j \in I} R(j)
$$

When the active failure of the component $i$ occurs and its relay protection refuses to action, which means that all of the breakers in the range of the set $I$ fall in the active failure, the path set cut off is described as the Eq.3.

$$
F(i)=\bigcup_{j \in I} S(j)
$$


The remained path set with any fault state can be find out by the path set $R(i), S(i)$ and $F(i)$. For example, to analyze consequence of the component $i$ with the $\mathrm{S}$ state, the component $\mathrm{j}$ with the $\mathrm{R}$ state, and the component $\mathrm{k}$ with the $\mathrm{M}$ state, firstly, the path set cut off should be solved out by the equation $S(i) \cup R(j) \cup R(k)$, and then remained path set can be solved out by the Eq.4.

$$
x=L \backslash[S(i) \cup R(j) \cup R(k)]=\overline{[S(i) \cup R(j) \cup R(k)]}
$$

The graph connectivity of the remained path sets is analyzed when a component with active failure. If is connected, the generators and transmission lines in the connected graph is analyzed. If the generator or transmission line is in the connected graph, it shows that it is not affected by the failure component. Otherwise the component is falling in forced outage. If the remained components do not constitute a connected graph, then the components of each and determine is analyzed to judge whether the components in the connected blocks are affected by the failure component.

\section{Evaluation Method Considering the Active Failure}

In this paper, two methods are used to consider the effect of the active failure when the Monte Carlo simulation method is used to evaluate the power system reliability. They are the amending parameter method and the simulating failure method.

The Amending Parameter Method. The amending parameter method is to correct the system component parameters by transforming the effect of the active failure. Firstly the active failure states are enumerated, the failure probability of the system components caused by the active failure is calculated, and then corrected to the failure probability of the component itself. Finally, the modified system components are evaluated by the Monte Carlo simulation method.

The and-order and below 3rd-order fault states are enumerated to analyze the active failure with the analytical method. The single fault states include R state, M state, S state and F state mentioned above. The fault states to be enumerated are the state of the single faults and the combinations of single faults.

The Simulating Failure Method. Considering the characteristics of each component above mentioned, the active failure of the components sampled with the simulating failure method, can be unified described as Eq.5, Eq.6, Eq. 7 and Eq. 8.

$$
\begin{gathered}
0<x \leq P L_{i} \\
P L_{i}<x \leq P L_{i}+P S_{i}, \\
P L_{i}+P S_{i}<x \leq P L_{i}+P S_{i}+P M_{i} \\
P L_{i}+P S_{i}+P M_{i}<x \leq P L_{i}+P S_{i}+P M_{i}+P R_{i}
\end{gathered}
$$

In the equations, $P L_{i}$ represents the probability of relay protection in the refusing action, ${ }^{P S_{i}}$ represents the probability of the component in short circuit fault, ${ }^{P M_{i}}$ represents the probability of the component in maintenance the state. $P R_{i}$ represents the probability of the component in fault state.

It is directly perceived to analyze the effect of the active failure on the system reliability by the simulating failure method. The active failure state is analyzed whether it causes the system components outage. If it is, regard the system components with outage as a fault.

Algorithm Explanation. The algorithm is explained by the following steps.

Step 1: Read system parameters.

Step 2: Set the maximum sampling number, use the system time as a random number seed initial, and then begin sampling.

Step 3: For the amending parameter method, enumerate the and-order and below 3rd-order fault states, analyze each fault state whether it causes the system components outage. If it is, record the system components outage probability, and go to Step 4. If not, go to Step 5. For the simulating failure method, sample the active failure of the components concluding the bus, breakers and relay 
protection, and then analyze the active failure state whether it causes the system components outage. If it is, regard the system components with outage as a fault, and the go to Step 5. If not, go directly to Step 5.

Step 4: Correct the system components outage probability caused by the active failure to the primitive parameters of the corresponding system components.

Step 5: Sample the elements of the system, judge and evaluate the connectivity of the system in the sampling state. Then calculate the power flow and use LINDO programming for overload correction.

Step 6: Judge the sampling number whether it is bigger than the maximum sampling number. If it is, go to Step 7. If not, go to Step 4 for the analytical method, and go to step 3 for the Monte Carlo simulation method.

Step 7: Calculate the reliability index including LOLP, EENS, and etc..

\section{Analysis of Example}

The IEEE RTS example system reliability is evaluated by the methods mentioned above. The system wiring is described in reference 7 and the IEEE RTS substation main wiring is described in reference 8 . The maximum sampling number is 100000 .

Comparison of Comprehensive Indexes. The calculation results are shown in Table 1.

Table 1 Comparison of comprehensive indexes

\begin{tabular}{|c|c|c|c|}
\hline \multicolumn{2}{|c|}{ method } & LOLP & EENS (MWh) \\
\hline \multicolumn{2}{|c|}{ Without active failure } & 0.084791 & 129208.23 \\
\hline \multirow{2}{*}{$\begin{array}{c}\text { With active } \\
\text { failure }\end{array}$} & Amending parameter method & 0.093784 & 142621.54 \\
\cline { 2 - 4 } & Simulating failure method & 0.110497 & 172800.09 \\
\hline
\end{tabular}

The reliability indexes considering the effect of the active failure are much higher. It proves that the effect of the active failure is necessary to the system reliability evaluation. Further more, the indexes are higher $30.32 \%$ and $33.74 \%$ by the amending parameter method and the simulating failure method than the method without considering the active failure. The simulating failure method can detect the high-order fault states and the common mode faults which are regarded as the cause of the blackout. The indexes are closer to the real by the simulating failure method.

Analysis of the Most Serious Fault State. The most important purpose of considering the active failure is to analyze the cause of the most serious fault state. The most serious fault states are enumerated in Table 2.

Table 2 the most serious fault state

\begin{tabular}{|c|c|c|c|c|}
\hline \multicolumn{2}{|c|}{ Method } & Components outage & Causes of outage & $\begin{array}{c}\text { Curtailment } \\
\text { of the load } \\
\text { (MW) }\end{array}$ \\
\hline \multicolumn{2}{|c|}{ Without active failure } & $\begin{array}{c}\text { Generators24,27,28,30,31,3 } \\
2\end{array}$ & Independent failure & 1144 \\
\hline \multirow{2}{*}{$\begin{array}{c}\text { With } \\
\text { active } \\
\text { failure }\end{array}$} & $\begin{array}{c}\text { Amending } \\
\text { parameter } \\
\text { method }\end{array}$ & $\begin{array}{c}\text { Generators8,20,24,27,28,30, } \\
31,32\end{array}$ & Independent failure & 1264 \\
\cline { 2 - 5 } & $\begin{array}{c}\text { Simulating } \\
\text { failure method }\end{array}$ & $\begin{array}{c}\text { Generators20,24,25,26,27,2 } \\
8,2932 \text { and lines 31 and 38 }\end{array}$ & $\begin{array}{c}\text { Relay protection } \\
\text { refusing action and } \\
\text { Independent failure }\end{array}$ & 1301 \\
\hline
\end{tabular}

The consequence of the most serious fault state is more serious analyzed by the metod with considering the active failure than that without considering. The curtailment of the loads is $10.49 \%$ and $13.72 \%$ higher. Especially, the blackout can be detected by the simulating failure method. When 
the relay protection of the breaker on node 22 refuses to action, six generators of the system and two lines fall in outage at the same time. The consequence is serious. The effective measures can be taken to avoid the blackout or reduce the blackout effect with the reference of the analysis result.

Analysis of the System Weaknesses. To analyze the effect of the active failure on the system reliability, the weaknesses, such as the curtailment of the load quantity and the number of the line overload, will be calculated.

The curtailment of the load quantity with considering the effect of the active failure is also much higher than those without considering, and with only some exceptions, it is also higher by the simulating failure method than the amending parameter method especially from node 3 to node 10 shown in Table 3.

Take the node 9 and node 10 for example. The main wiring of the node 9 is angle scheme, which use a breaker connecting the two angles. If the active failure of the breaker occurs, the whole substation will be in outage. The components of the system corresponding to the nodes will be in outage, too. So the curtailment of the load quantity will greatly increase.

With considering the effect of the active failure, the number of the line overload is significantly increased, especially for Lines 3, 4, 5, 8, 9, 10, 12, 13. The result is shown in Fig.2.

Table 3 the curtailment of the load quantity

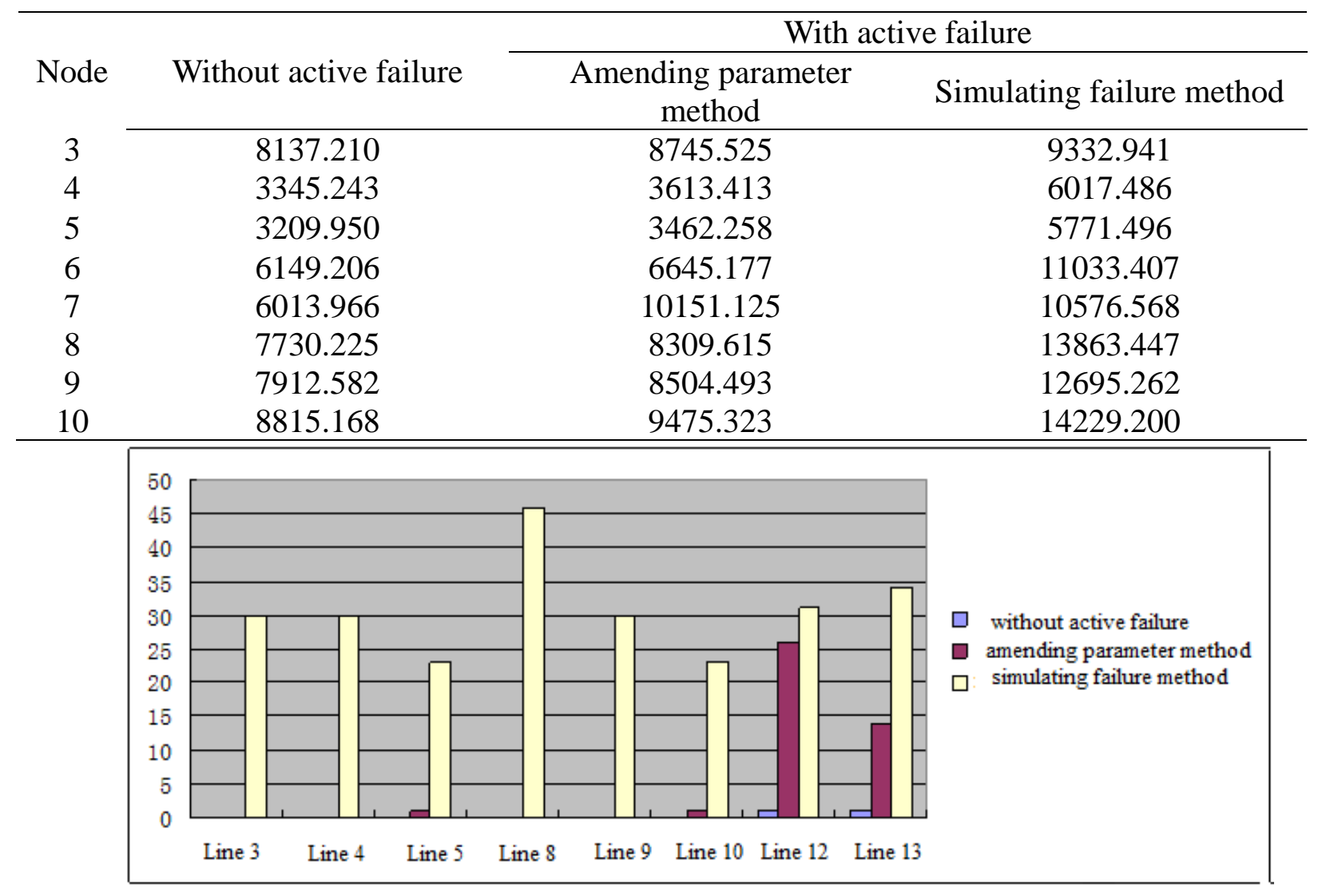

Fig. 2 the number of the line overload

To increase the lines capacity, or to adopt the main wring with high reliability, will be effective to reduce the lines overload and reduce the curtailment of the load quantity.

\section{Conclusion}

In this paper, the effect of the active failure on the power system reliability is considered which is more precise to evaluate the power system reliability. Two methods are used to evaluate the effect of the active failure on the power system. The results of the analysis of example prove the necessity 
of considering the effect of the active failure on the system reliability, and the amending parameter method is more significant and closer to the real.

From the analysis, the amending parameter method is better to find out the most serious fault state and detect the weaknesses than the amending parameter method and the method without considering the active failure. It can help the run planning personnel taking measures to increase the system reliability.

\section{References}

[1]Deqiang Gan, Hu Jiangyi,Han Zhenxiang,Thinking on several blackouts in the world in 2003,Automation of electric power systems Journal ,2004,28(3).

[2]Baosheng Tang, The blackout area of London and its lessons, Grid Technology Journal,2003,27(11).

[3]Grover M S, Billinton. R. A Computerized Approach to Substation and Switching Station Reliability Evaluation. IEEE Trans. on PAS[J]. 1974, 93: 1488-1497.

[4]Singh C, Patton A D. Models and Concepts for Power System reliability Evaluation Including Protection-System Failures. Electric Power \& Energy Systems[J]. 1981， 100 (2): 608- 617.

[5]Billinton. R, Tatla J. Composite Generation and Transmission System Adequacy Evaluation Including Protection System Failure Mode. IEEE Trans. on PAS[J]. 1983. 102.

[6]Xifan Wang, Research on the reliability of power station main wiring(1)-The Basic model and algorithm, Xi’an Jiao Tong University Journal,1990,23.

[7]IEEE Committee Report. IEEE Reliability Test System. IEEE Transactions on Power Apparatus and System,1979,98(6).

[8]The IEEE Reliability Test System-1996, IEEE Transaction on Power System,1999,14(3). 